\title{
Les Conventions climat et biodiversité : une nouvelle géopolitique des rapports de force
}

Un an après les grands engagements internationaux de 2015, qui avaient montré un alignement exceptionnel des anticipations et des plans des acteurs gouvernementaux de toute la planète, ainsi que des acteurs privés et de la société civile, les cycles politiques ont repris leur droit, annonçant des résistances dures aux changements qui semblaient consensuels il y a un an.

Les résultats des conférences des conventions internationales sur le climat (COP 22 de Marrakech) et sur la biodiversité (COP 13 de Cancún) tenues fin 2016 témoignent de ces résistances. Plus que jamais les relations hommes-natures au cœur des réflexions de NSS nous permettent de rendre compte des bouleversements géopolitiques et technologiques à l'œuvre.

L'élection de Donald Trump a singulièrement troublé les débats de la COP 22 en faisant revivre, 15 ans après, l'épisode du refus des États-Unis de s'associer au protocole de Kyoto dans cette même ville de Marrakech. Mais si le nouveau président américain a le pouvoir de s'opposer aux régulations en faveur de la transition énergétique, pourra-t-il vraiment relancer le charbon et la sidérurgie, si cela n'a aucun sens économique et va à l'encontre des exhortations des grandes entreprises pour alimenter l'économie américaine avec des énergies neutres en carbone ? Pourra-t-il empêcher la Californie ou d'autres États fédérés, ou les acteurs économiques de nombreux secteurs, de remplir les engagements à long terme qu'ils se sont fixés? A-t-il le pouvoir de changer les anticipations du secteur financier vis-à-vis des énergies fossiles, considérées comme des investissements sans avenir? Même si la plupart des acteurs réunis à Marrakech ont maintenu leur engagement en faveur d'une transformation profonde de nos sociétés et de nos économies, le nouveau président américain a en main les leviers pour aller vers de tels rapports de forces, comme il le fait visiblement avec la Chine.

Le lien entre la libéralisation des échanges commerciaux et la consolidation de la paix qui était au cœur du projet européen et du traité de Rome est aujourd'hui remis en cause partout dans le monde. On en voit trace dans les atermoiements autour de l'accord de partenariat transpacifique mené par les États-Unis, du CETA entre l'Union européenne et le Canada, du TTIP entre l'Union européenne et les États-Unis. Seule la Chine continue d'afficher son projet d'expansion des échanges avec les nouvelles routes de la soie (One Belt One Road). En recentrant son industrie sur la consommation intérieure, elle doit en effet réduire sa dépendance aux exportations de matériaux intensifs en énergie fossile, tout en exportant infrastructures et technologies pour le développement durable, par exemple le photovoltaïque, vers l'Afrique et l'Asie du Sud.

La Chine a-t-elle pour autant le pouvoir et le souhait de s'imposer comme leader dans l'action climatique et de porter seule ce projet de mondialisation auquel s'opposent de plus en plus de gouvernements, notamment dans les pays développés, portés par les classes moyennes qui ont souffert des impacts de la mondialisation récente?

Indispensable pour rendre crédible l'accord de Paris, l'engagement des acteurs non étatiques pour l'action climatique a fortement marqué Marrakech, où les États ont pu sembler plus en retrait. De nombreuses coalitions et alliances se sont déclarées, au point que leur enregistrement et leur suivi posent problème au secrétariat de la Convention Climat. La plateforme NAZCA (Non-State Actor Zone for Climate Action) recense plus de 10000 initiatives des acteurs privés et des collectivités. Dans ce foisonnement, on trouve de nombreux mouvements sociaux, qui ont réclamé la subsidiarité et une forme de souveraineté pour agir et transformer le monde depuis les niches d'innovation locale. Ils courent aujourd'hui le risque de se retrouver liés aux mouvements politiques nationalistes, de voir leur revendication de souveraineté brouillée par un repli sur les frontières et sur des identités, dans un contexte, depuis l'invasion de l'Irak jusqu'à la guerre en Syrie, d'exacerbation des nationalismes.

Il importe toujours plus de construire des coalitions pour le changement depuis le niveau local jusqu'au 
niveau européen et international, pour défendre d'autres projets d'abolition des frontières, d'autres projets de transition et de transformation, d'autres projets entre pays européens, d'autres relations entre l'Europe et le reste du monde. C'est d'autant plus urgent que l'affirmation de la force se fera de plus en plus arrogante et sans fard, pour maintenir les intérêts en place et préserver les rentes sur les énergies fossiles. L'accaparement de la valeur est en effet l'enjeu majeur de nos modèles de développement au sein desquels sont liés impacts sociaux et environnementaux toujours plus graves.

Toutes les batailles sont importantes, dans cette époque d'affirmation des relations géopolitiques à toutes les échelles, pour construire de nouveaux projets de biens communs locaux et mondiaux. La lecture des rapports de force est nécessaire pour comprendre la période actuelle, pour identifier où réside le pouvoir de transformer les sociétés et pour agir en conséquence. Les forums internationaux comme la Convention sur la diversité biologique, jouent aussi un rôle majeur dans la construction de coalitions des échelles locales à l'échelle mondiale. Souvent dédaignée au profit des négociations sur le climat, parce qu'elle est jugée incapable de peser sur les décisions nationales, cette Convention est le lieu $d$ 'affrontements et d'alliances qui se nouent et progressent de manière beaucoup moins visible.

Ainsi, lors d'une conférence de cette convention, passée largement inaperçue en décembre dernier (la COP 13 de Cancún), on a pu prendre la mesure des transformations de nos sociétés et de notre monde au travers des innovations numériques et biotechnologiques qui changent à la fois nos relations à notre environnement et la répartition de la valeur créée par sa mise en exploitation. Deux thèmes de travail se sont rejoints : celui de la biosécurité (qui évalue les risques de la biologie synthétique) et celui du mécanisme mondial multilatéral de partage des avantages relevant du protocole de Nagoya. On prend conscience que l'accès physique à la plante n'est plus nécessaire pour la recherche sur le vivant, $d u$ fait $d u$ développement des données séquentielles numériques (la bio-informatique) et des nouvelles techniques de modification du génome qui échappent à la fois au contrôle démocratique sur la manipulation du vivant et aux règles d'accès et de partage des avantages tirés de l'exploitation des ressources génétiques et des connaissances traditionnelles (APA). 150 ONG ont demandé un moratoire sur le «forçage de gènes ". La COP 13 s'est ainsi attaquée à des enjeux, tout à fait essentiels, d'accaparement de la valeur, mais aussi de pratiques d'appropriation et de manipulation du vivant. Si les représentants des peuples autochtones ont beaucoup investi la scène de la Convention sur la diversité biologique, c'est qu'elle permet d'alerter aussi sur le risque d'écrasement de la diversité lié aux transformations du monde. Qu'elles soient intentionnelles comme celles appelées par les engagements de 2015 ou davantage subies comme la révolution numérique, ces évolutions ne devraient pas conduire à homogénéiser et standardiser, mais au contraire à préserver, autant pour des raisons morales que pour leur valeur d'option, non seulement la diversité biologique et culturelle, mais aussi la capacité d'agir de la diversité des communautés humaines. Dans un avenir hautement incertain, chacune est porteuse d'une partie des solutions de l'agenda du développement durable.

Dans cette période d'affirmation et de confrontation des puissances, il serait tentant de concentrer les stratégies de changement sur le basculement massif de grands secteurs (industrie, transports, finance...) vers l'économie décarbonée. Réussir la transformation vers le développement durable impose cependant tout autant de redonner du sens à un projet mondial où les communautés locales et les mouvements sociaux, dans toute leur diversité, ont aussi le pouvoir de changer le monde. Un chantier auquel il est indispensable que participent les recherches des diverses disciplines, dans un rôle de critique ou d'accompagnement, au plus près du local comme à l'échelle mondiale.

Sébastien Treyer et Catherine Aubertin 